\title{
A Clinical Report on Association of Hypothyroidism and Hyperprolactinemia with Female Fertility
}

\author{
Seema Rai*, Shonali Chhatar, Shubhada Kalvit and Pravin Kalvit \\ Department of Zoology, Guru Ghasidas Vishawishvavidyalaya, Koni, Bilaspur, India \\ *Corresponding Author: Seema Rai, Department of Zoology, Guru Ghasidas \\ Vishawishvavidyalaya, Koni, Bilaspur, India.
}

Received: June 19, 2021

Published: July 13, 2021

(C) All rights are reserved by Seema Rai., et al.

\begin{abstract}
Introduction: Hypothyroidism is a common thyroid disorder. It affects people all over the world irrespective of age, sex, race, and level of wealth and education. Prevalence of hypothyroidism is $2-4 \%$ in women in their reproductive age groups. Hypothyroidism can affect fertility due to anovulatory cycles, luteal phase defects, hyperprolactinemia and sex hormone imbalances. The objective of present study is to do an assessment regarding the prevalence of clinical/sub-clinical hypothyroidism in infertile women and the treatment responses for hypothyroidism on infertility.

Methods: A total of 120 infertile women were found who, visited, the infertility clinic for the first time. Reports of clinical parameter such as obtained for, thyroid stimulating hormone (TSH), prolactin (PRL) and estrogen ( $\mathrm{E}_{2}$ ). Analysis were done at early stage of infertility checkup, instead of straight away going for more expensive tests or invasive procedures. Results: Finding, suggest an increased level of TSH, PRL AND E2 in infertile women of middle age (30 - 40 yrs).

Conclusion: Identification of hypothyroidism and hyperprolactinemia during early and late reproductive stage stages can be preventive in infertile females hence such screening should be mandatory in the primary phage of such females.
\end{abstract}

Keywords: Thyroid; Hypothyroidism; Hyperprolactinemia; Fertility

\section{Introduction}

Approximately 15 to $20 \%$ of the population of couple globally are affected with the problem of infertility. The abnormal function of hypothalamic-pituitary-ovarian (HPO) axis appears to be the most common female factors. Serum prolactin and thyroid stimulating hormone (TSH) has always been considered to play the important role in regulation of fertility in women. Thyroid dysfunction can interfere with ovulation which can impair fertility [1]. Hypo or and hyperthyroidism as well as subclinical thyroid dysfunction are directly associated with reproductive cycles, anovulation, decrement in fecundity and increment in complaint of pregnancy [2]. Triiodothyronine (T3) in combination with follicle stimulating hormone (FSH) stimulates the differentiation of gran- ulosa cells by suppressing its apoptosis via protein kinase pathway [3]. Literature suggest that thyroid stimulating hormone has stimulatory effect on leukemia inhibitory factor (LIF) expression of endometrial cell culture hence might have involvement in the process of implantation [4].

Further, prolactin a hormone promotes is not only produced by the uterine cells but it is also produced by immune cells, brain, breasts, prostate, skin, and adipose tissues. Hypothalamic dopamine regulates production of prolactin. If there is more dopamine then there will be less prolactin release. Hyperprolactinemia has a negative impact on the pulsatile secretion of gonadotropic releasing hormone (GnRH) [2]. Hyperprolactinemia can cause menstrual irregularities and impaired ovulation [5]. Estrogen also enhances 
the production and secretion of prolactin from the pituitary gland with a significant role in both reproductive as well as non-reproductive system. Estrogen are also essential for early development, embryonal and fatal development of the brain network. These hormones are very much important for the reproductive, metabolic and many other physiological activities.

An elevation of TRH, which causes an increase of prolactin along with TSH is caused by primary hypothyroidism [6]. High level of estrogen also enhances the rate of thyroid dysfunction. Estrogen dominance also causes hypothyroidism which leads to infertility.

\section{Aim of Study}

We aim a survey in order to make an assessment regarding the prevalence of clinical/sub-clinical hypothyroidism in infertile women of different reproductive age groups and the treatment responses for hypothyroidism on infertility.

\section{Materials and Methods}

Survey was made in the Kalvit Infertility Center situated in sipat chowk, Bilaspur to perform the study from $1^{\text {th }}$, February, 2020 to $20^{\text {th }}$, March, 2020. The hormonal reports of 120 infertile women were analyzed with the permission of the authority. For the accomplishment the aim of the present investigation, following objectives were observed:

- $\quad$ Case study of hormonal concentration of 120 infertile female.

- Thyroid stimulating hormone [TSH] in 120 infertile female.

- Prolactin hormone [PRL] in 120 infertile female.

- $\quad$ Estrogen $\left[\mathrm{E}_{2}\right]$ in 120 infertile female.

Those sample were categorized in 4 age groups starting from (25 - 30 years, 31 - 35 years, 36 - 40 years and 41 - 45 years). The analyzed hormones were TSH (Thyroid Stimulating Hormone), Estrogen and prolactin. The value of each patient with 5-year difference were pooled and average were taken to compare with normal range of hormone. The data were represented in the form of histogram and pie chart.

\section{Results}

A total of 120 infertile women enrolled for this study, $62.5 \%$ of infertile women have noted with raised level of TSH,
$68.5 \%$ infertile women had high level of estrogen whereas $60.5 \%$ women had increased prolactin level. The remaining 4-5\% of infertile women had raised TSH, estrogen and prolactin level which may be due to hypothalamic or any other pituitary disorder.

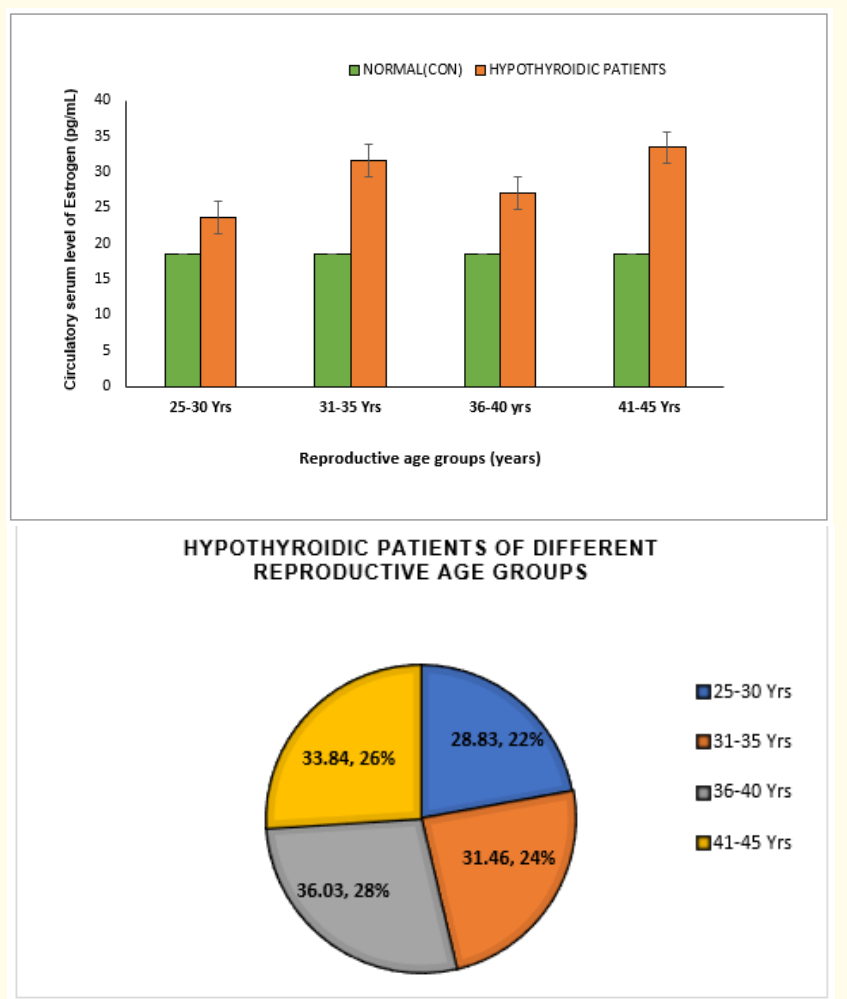

Figure 1: Variation in Circulatory level of Estrogen among infertile women of different age groups (upper panel) and percentage of the normal and hypothyroidic women (Pie diagram lower panel). Histogram represents Mean \pm SE.

Infertile women were further subdivided into subclinical $(4.00$ - 6.00 mili international units per liter) and clinical (> 6.00 mili international units per liter) hypothyroidism based on the TSH levels. It was found that most of the infertile women were with subclinical and clinical hypothyroidic women were less than subclinical [7]. (Method adopted from the study of Cooper, 1991). The mean of hormone TSH varies in different age group and highest value fall under the age group of 25 - 40 years. The mean of hormone estrogen also varies in different age group and the highest value comes under 30 - 40 years. The highest mean value of hormone prolactin falls under 35 - 40 years. 
Increased Estrogen level in infertile women in different age groups

- $66 \%$ infertile women are hypothyroidic between 25 - 30 years of reproductive age group.

- $\quad 68 \%$ infertile women are hypothyroidic between 31 - 35 years of reproductive age group.

- $\quad 71 \%$ infertile women are hypothyroidic between 36 - 40 years of reproductive age group.

- $\quad 69 \%$ infertile women are hypothyroidic between 41 - 45 years of reproductive age group.

Increased TSH level in infertile women in different age groups

- $48 \%$ infertile women of 25 - 30 years of reproductive age group were hypothyroidic.

- $68 \%$ infertile women of 31 - 35 years of reproductive age group were hypothyroidic.

- $\quad 67 \%$ infertility belonged with women of $36-40$ and $41-45$ years of reproductive age groups were hypothyroidic.

Increased prolactin level in infertile women in different age group

- $\quad 56 \%$ infertile women are hypothyroidic between 25 - 30 years of reproductive age group.

- $63 \%$ infertile women are hypothyroidic between 31 - 35 years of reproductive age group.

- $\quad 59 \%$ infertile women are hypothyroidic between 36 - 40 years of reproductive age group.

- $64 \%$ infertile women are hypothyroidic between 41 - 45 years of reproductive age group.

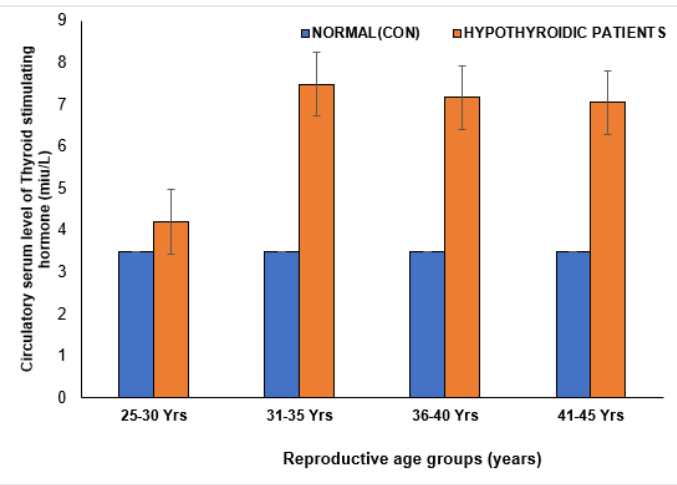
HYPOTHYROIDIC PATIENTS OF DIFFERENT REPRODUCTIVE AGE
GROUPS

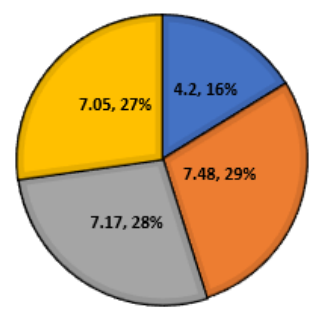

प25-30 Yrs

口31-35 Yrs

口36-40 Yrs

口41-45 Yrs

Figure 2: Variation in Circulatory level of thyroid stimulating hormone (TSH) among infertile women of different age group (Upper panel) and pie diagram showing percentage of the normal and hypothyroidic women (lower panel). Histogram represents

Mean \pm SE.

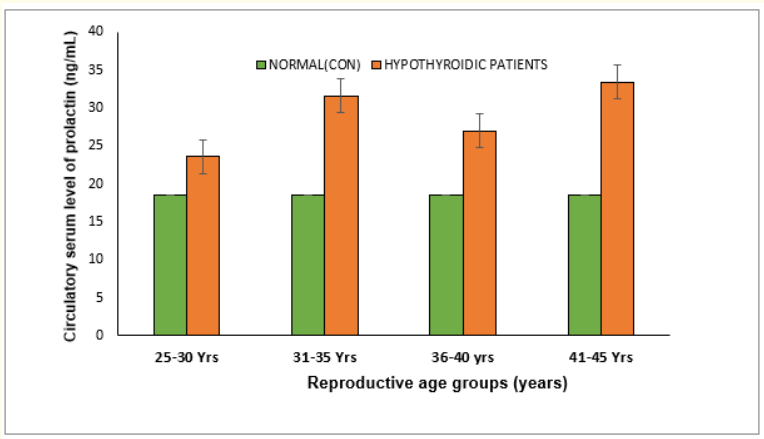

Hypothyroidic patients of different reproductive age groups

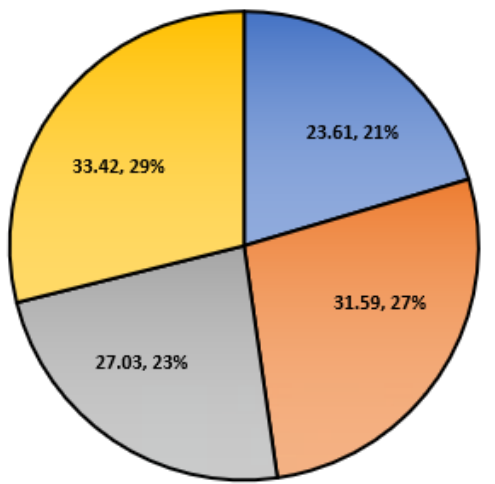

ם25-30 Yrs

口31-35 Yrs

口36-40 yrs

口41-45 Yrs

Figure 3: Right panel - Variation in Circulatory level of prolactin among infertile women of different age groups (upper panel). pie diagram showing percentage of the normal and hypothyroidic women (lower panel) Histogram represents Mean \pm SE. 


\section{Discussion}

Infertility is a global problem nowadays and it has effected people of all communities. Although the cause of infertility may be as per the variation in geographical location and socioeconomic position. Report suggest that out of 60 - 80 million infertile couple reported per year 15 - 20 million couples belong to India [8]. The thyroid hormones have great significant effects on reproduction and pregnancy. The thyroid disorders are involved in a broad spectrum of reproductive diseases, extending from abnormal sexual development to infertility and menstrual irregularities. Increased production of TRH is associated with hypothyroidism which is responsible for the stimulation of pituitary to secrete TSH and prolactin $[9,10]$. High level of prolactin(hyperprolactinemia) adversely influence fertility potential by reducing GnRH pulsatility and thereby ovarian function. Gynecologists generally check TSH and PRL levels in every infertile women [2], regardless of their menstrual rhythm. Hyperprolactinemia may result from stress or depression. The thyroid disorders being the common cause of infertility can be corrected by appropriate level of thyroid hormones. The normal TSH levels are necessary for fertilization. The data also says that variation in TSH levels in the broadline cases, i.e. 5 - 6 and $>6.0$ mili international units per liter, should not be ignored in infertile females which can be asymptomatic for clinical hypothyroidism. The data of this study shows that the middle-aged women are mostly affected.

Excess estrogen can also block the uptake of thyroid hormone, which leads to hypothyroidism. The normal range of estrogen (E2) level for menstruating women range from 15-350 picograms per millilitre $(\mathrm{pg} / \mathrm{mL})$. Postmenopausal women's normal $\mathrm{E}_{2}$ level should be lower than $10 \mathrm{pg} / \mathrm{mL}$. For the regulation and control of female body estrogen is needed. It effects our menstrual cycle and fertility to our memory and skin. Its level also fluctuates over time and halts once menopause hits. High level of estrogen in the body system is called estrogen dominance. It gives impact on thyroid health by stopping the conversion of T4 into T3 thyroid hormone which leads to low T3 levels. Too much estrogen blocks the uptake of thyroid hormone. It shows symptoms like weight gain, fatigue, infertility, mood swings, slow metabolism, irregular periods, polycystic ovaries (PCOS).

High level of prolactin is known as hyperprolactinemia. Hypothyroidism is associated with low thyroid level and raised prolactin level. It is implicated in ovulatory dysfunction that leads to infertil- ity. Prevalence of hyperprolactinemia is subclinical hypothyroidism. Hyperprolactinemia was defined as elevated serum PRL (> $19.5 \mathrm{ng} / \mathrm{mL}$ ). Normal prolactin level in pregnant women: 80 to 400 $\mathrm{ng} / \mathrm{mL}$. Normal prolactin level in non-pregnant women: less than $25 \mathrm{ng} / \mathrm{mL}$. Additionally, patients with higher TSH value have significantly higher level of prolactin. This means severe hypothyroidism displayed by higher TSH levels, leads to a greater thyrotropinreleasing-hormone -mediated prolactin release. The prevalence of infertility was higher in the presence of high prolactin level than in the presence of normal prolactin level in patients with mild hypothyroidism.

Increased level of TRH causes rise in PRL, and these patients may suffer from galactorrhea. This study was experimented on 120 women of age group 25 - 50 years on their visit to an infertility clinic of Gynecology and Obstetrics (Kalvit Infertility Center, Bilaspur) from January to March 2020. Routine investigation such as blood tests, T3 and T4 tests, TSH tests, hyperprolactinemia tests and ultrasounds (when required) were done. The normal level of TSH, estrogen and prolactin are 0.5-3.0 mili international units per liter, 15-350 picograms per mili liter and 1.3 - 25.0 nanogram per mili liter. Therefore, from this study hypothyroidism was considered at TSH level of $>4.2$ mili international units per liter and prolactin level of $>25$ nanogram per mili liter. Treatment was given to hypothyroid infertile females which depends on TSH levels. Statistical analysis of the results are done by the help of percentage.

Hypothyroidism is a common thyroid disease. It affects people all over the world - of every age, sex, race, and level of wealth and education. Prevalence of hypothyroidism is $2-4 \%$ in women in their reproductive age groups. Hypothyroidism can affect fertility due to anovulatory cycles, luteal phase defects, hyperprolactinemia and sex hormone imbalances. To study prevalence of clinical/subclinical hypothyroidism in infertile women and the treatment responses for hypothyroidism on infertility. A total of 120 infertile women visiting the infertility clinic for the first time were investigated for thyroid stimulating hormone (TSH), prolactin (PRL) and estrogen $\left(\mathrm{E}_{2}\right)$. Measurement of TSH and PRL should be done at early stage of infertility checkup instead of straight away going for more expensive tests or invasive procedures. Simple, oral treatment of hypothyroidism for 3 months to 1 year can be of significant benefit to conceive in otherwise asymptomatic infertile women. 


\section{Conclusion}

The findings suggest that women with primary infertility have significant alterations and impairment not only in hypothalamicpituitary-ovarian axis but circulatory prolactin level equally participates for the various case of infertility. There is not only a single hormone which is involved in fertility but a fine tuning between female gonadal hormone estrogen, pituitary hormone TSH and the prolactin as well is responsible to maintain the normal fertility among the women, irrespective of the age group. It is seen that the increasing level of estrogen, TSH and prolactin in the bloodstream of infertile women cause hypothyroidism. Present data showed that the incidence of hyperprolactinaemia in infertile women population was significantly high in percentage than to hypothyroidic women. Therefore, is may be inferred that long term hypothyroidism and hyperprolactinemia together or alone may lead to ovarian dysfunction among the middle-aged reproductive females (35 - 40 years).

\section{Bibliography}

1. Moon Kyoung Cho., et al. "Thyroid dysfunction and subfertility". Clinical and Experimental Reproductive Medicine 42 (2015): 131-135.

2. Hymavathi $\mathrm{K}$ et al. "Correlation of serum thyroid hormones and prolactin levels to female infertility". International Journal of Reproduction, Contraception, Obstetrics and Gynecology 5 (2016): 4018-4024.

3. Zhang C., et al. "Effects of 3, 5, 3'-triiodothyronine ( $\mathrm{t} 3$ ) and follicle stimulating hormone on apoptosis and proliferation of rat ovarian granulosa cells". The Chinese Journal of Physiology 56 (2013): 298-305.

4. Aghajanova L., et al. "Thyroid-stimulating hormone receptor and thyroid hormone receptors are involved in human endometrial physiology". Fertility and Sterility 95 (2011): 230-237. 237.e1-237.e2.

5. Mishra R., et al. "Prolactin levels in infertility with menstrual irregularities". Journal of Obstetrics and Gynaecology 52 (2002): 40-43.

6. Prateek Shukla., et al. "Pituitary Hyperplasia in Severe Primary Hypothyroidism: A Case Report and Review of the Literature". Hindawi Case Reports in Endocrinology (2019): 7.
7. Cooper DS. "Subclinical hypothyroidism". Advances in Endocrinology and Metabolism 2 (1991): 77.

8. Sudha G., et al. "Causes of female infertility: A cross-sectional study". International Journal of Latest Research in Science and Technology 2 (2013): 119-123.

9. Shamali J., et al. "Hyperprolactinemia and Its Comparison with Hypothyroidism in Infertile Women". International Journal of Recent Trends in Science and Technology 8 (2013): 106-108.

10. Avasthi K., et al. "Hyperprolactinema and its correlation with hypothyroidism in infertile women". Journal of Obstetrics and Gynecology of India 56 (2016): 68-71.

\section{Volume 5 Issue 8 August 2021}

(C) All rights are reserved by Seema Rai., et al. 